\title{
Discrete or Continuum? It Matters for Cells
}

\section{A new model shows that the properties of waves produced in a cell-signaling process strongly depend on whether the cells are considered to be discrete entities or a collective mass.}

\section{By Katherine Wright}

C ut your finger, and thousands of white blood cells called neutrophils swarm to the wound to fight bacteria. Captivated by microscopy images of this migration, Paul Dieterle and Ariel Amir of Harvard University set out to better understand the process. Their previous model, and those of others, assumed that the cells behaved as a continuum. Now the duo has looked at how their model's predictions change if the cells are instead treated as separate entities [1]. They find that the dynamics of the system can change significantly, producing unexpected behaviors.

Neutrophils are thought to communicate with each other by releasing diffusible molecules that move through the surrounding fluid as a wave. While most models treat these waves as collective emanations, Dieterle and Amir consider waves produced by a population of discrete cells. The emission starts with one cell emitting a diffusible molecule. After a given time period, those molecules reach a neighboring cell, causing

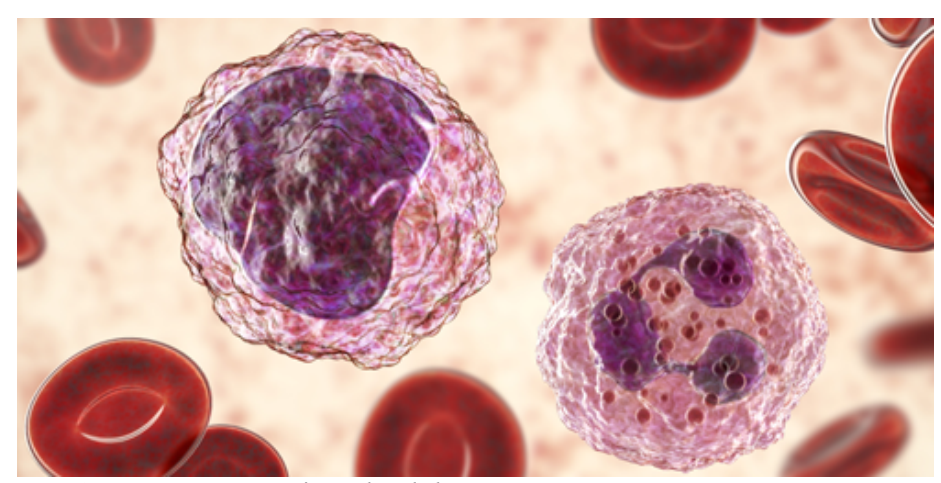

Credit: Kateryna ${ }_{K}$ on/stock.adobe.com it to emit the same molecules. The process then repeats, building up a wave of molecules that moves through the fluid.

The duo finds that the speed of the wave depends on a number of parameters, including the cell density and the dimension of the system. For example, they find that for a sparsely populated 1D system, in which the cells respond to molecular concentrations above a threshold, the waves move slower than predicted by continuum models. Increasing the cell density causes the speed disparity to disappear. Waves also move more slowly for 2D, but the disparity persists, while in 3D, the opposite occurs-the wave speed matches the continuum one for sparsely arranged cells and gets slower as density increases.

Katherine Wright is the Deputy Editor of Physics.

\section{REFERENCES}

1. P. Dieterle and A. Amir, "Diffusive wave dynamics beyond the continuum limit," Phys. Rev. E 104, 014406 (2021). 